\title{
Domenico Gerosolimitano a Venezia
}

\author{
Pier Cesare IOLY ZORATTINI \\ Università degli Studi di Udine
}

\begin{abstract}
Il primo di luglio del 1593 si presentava alla Casa dei Catecumeni di Venezia ${ }^{1}$, per chiedere di essere battezzato, Samuel Vivas, un «hebreo levantin de Gierusalem chiamato chaccham, che in italiano dicemo dottor ${ }^{2}$. La Congregazione lo accolse e, il 29 dello stesso mese, da un'altra annotazione dei Catecumeni ${ }^{3}$, si apprende che erano stati incaricati l'arcivescovo di Spalato, Girolamo Corner, Giovan Andrea Venier ${ }^{4}$ e Marco Scotto di trovare i padrini per tenerlo a battesimo. A quest'ultimo infine e a pre' Francesco di San Giacomo dell'Orio ${ }^{5}$ sarebbe toccato il compito di provvedere alla vestizione del Vivas. Una settimana dopo, il 6 agosto 1593, Samuel, all'età di 38 anni $^{6}$, riceveva solennemente il battesimo a
\end{abstract}

${ }^{1}$ Cfr. B. Pullan, The Jews of Europe and the Inquisition of Venice, 1550-1670 (Oxford 1983; trad ital. Roma 1985) p. 335.

2 Vid. infra Appendice Documentaria 14v.

${ }^{3}$ Cfr. Archivio delle Istituzioni di Ricovero e di Educazione di Venezia [AIRE], Catecumeni, CAT B 4, «Notatorio delle parti, 1592-1599» c. 22r.

${ }^{4}$ Potrebbe trattarsi di Zuan Andrea Venier (28 febbraio 1551 - 7 agosto 1604) di Gerolamo, che fu capitano a Bergamo. Cfr. M. Barbaro, Arbori de' patritii veneti, Archivio di Stato di Venezia [ASV], Miscellanea Codici I: «Storia Veneta» 17-23, VII, f. 237.

${ }^{5}$ Con tutta probabilità identificabile con pre' Francesco Mazza, a quel tempo prete titolato (cfr. Archivio della parrocchia di S. Giacomo dell'Orio di Venezia, Catastico o sia miscellane delle notizie principali e più necessarie della chiesa parrochiale e collegiata di San Giacomo dell 'Orio raccolte dal ... pre' Girolamo Catane pievano, tomi 3, i, p. 195) e in seguito parroco di San Giacomo dell'Orio dal 1606 al 1618 (cfr. A. Niero, Chiesa di San Giacomo dall'Orio [Venezia 1990]), deceduto in detta parrocchia l'11 febbraio 1619. (Cfr. Archivio della Parrocchia di S. Giacomo dell'Orio, «Morti 1601 sino 1631», cc. n.n.)

${ }^{6}$ Cfr. Archivio Storico del Patriarcato di Venezia [ASPV], Battesimi dei Catecumeni, reg. 1, «Battesimi principia 1590, 3 marzo finisse 1594 ultimo marzo» cc. $14 \mathrm{v}-15 \mathrm{r}$. 
S. Giovanni e Paolo, la chiesa dei Domenicani, dal patriarca di Venezia Lorenzo Priuli ${ }^{7}$, illustri padrini gli furono l'arcivescovo di Spalato ${ }^{8}$ e Alvise Bollani ${ }^{9}$ vescovo di Milopotamo. Al neofito venne imposto il nome di Domenico, presumibilmente in onore del santo patrono dell'ordine domenicano il cui natale cade appunto in quel giorno ${ }^{10}$.

L'8 settembre, quindi, Domenico riceveva la cresima in Patriarcato e in tale circostanza gli era padrino Girolamo Corner, a quel tempo «governator» della Casa dei Catecumeni di Venezia ${ }^{11}$. Ricordiamo infine che, subito dopo il battesimo, il 12 agosto 1593, la Congregazione aveva incaricato due «fratelli», il «pioan di San Mauricio» ${ }^{12}$ e ser Domenego Marin ${ }^{13}$, di fare istanza in Collegio affinché gli venisse corrisposta «qualche cossa» ${ }^{14}$, segno indubbio delle sue misere condizioni economiche.

La rilevanza della figura di Domenico, l'anno della conversione, cioè 1593 , l'età di 38 anni che ne situa la nascita verso il 1555 , la

${ }^{7}$ Lorenzo Priuli fu patriarca di Venezia dal 1590 al 1600, anno della morte. Cfr. A. Niero, I patriarchi di Venezia (Venezia 1961) pp. 99-106.

8 Arcivescovo di Spalato dal 1575 o 1577 (?) al 1602, anno della morte, fu il domenicano Giovanni Domenico Marco o Marcot. Cfr. P. B. GAMs, Series Episcoporum Ecclesiae Catholicae (Ratisbonae, Typis et sumptibus Georgii Josephi Manz 1873) p. 421; C. Eubel, Hierarchia Catholica Medii et Recentioris Aevi, 8 voll. (Monasterii 1913-1978 [2]) vol. III p. 303.

9 Vescovo di Milopotamo in Candia dal 1593 (?) fino al 19 gennaio 1594 fu il crocifero Alvise Bollani. Cfr. ASV, Miscellanea Codici III serie (Codici Soranzo), reg. 31; ASV, G. A. Cappellari Vivaro, Famiglie Venete, ms. I, p. 466; Eubel Hierarchia vol. IV p. 242. Una prova della sua presenza a Venezia in quei mesi è il suo ringraziamento, il 30 ottobre 1593 , a papa Clemente VIII per avergli concesso il suddetto vescovato. Cfr. ASV, Senato deliberazioni Roma ordinaria, reg. 9, c. 171v (antica numerazione). Ringrazio la dott. Maria Pia Pedani Fabris per la preziosa collaborazione.

${ }^{10}$ Domenico di Guzman morì a Bologna il 6 agosto 1221.

11 Girolamo Corner fu governatore dei Catecumeni dal 19 marzo 1592 al 24 novembre 1594. Cfr. AIRE, Catecumeni, CAT B 4, «Notatorio delle parti, 1592 1599» cc. $2 \mathrm{v}, 41 \mathrm{v}$. Identificabile forse con quel Girolamo Corner (1562-1634) di Giorgio; del ramo di S. Cassan in calle della Regina, cavaliere e procuratore di S. Marco, che, tra l'altro, fu ambasciatore della Repubblica presso papa Urbano VIII. Cfr. Barbaro Arbori vol. III f. 55; R. Derosas, Dizionario Biografico degli Italiani, 47 voll. (Roma 1960-1997) vol. 29 pp. 236-241, s.v. Ringrazio sentitamente il prof. Giuseppe Ellero, direttore dell AIRE, per la gentile collaborazione.

${ }_{12}$ Dal 1571 al 1595 fu parroco di S. Maurizio a Venezia Nicolò Fausto, canonico di S. Marco e maestro delle cerimonie. Cfr. F. Corner, Ecclesiae Venetae antiquis monumentis ..., 18 tomi rilegati in 9 voll. (Venetiis, typis Joannis Baptistae Pasquali 1749) vol. III, quintae decadis, p. 382.

${ }_{13}$ Potrebbe trattarsi di Domenico Marin (15 agosto 1566 - 6 dicembre 1617) di Michiel che fu «avvocato de prigioni». Cfr. Barbaro Arbori vol. IV f. 502.

${ }^{14}$ Cfr. AIRE, Catecumeni, CAT B 4, c. 23r. 
sua qualifica di rabbino e infine la provenienza gerosolimitana coincidono con i dati a nostra disposizione concernenti un noto neofito e censore della stampa ebraica operante a Venezia e in Italia tra la fine del '500 e il primo ventennio del '600: Domenico Gerosolimitano.

La data della conversione del Gerosolimitano è stata oggetto di un dibattito ${ }^{15}$, ma già nel 1903 Ignazio Guidi la fissava al 1593 sulla base di un' autobiografia del Nostro contenuta in un testo autografo conservato presso l'Archivio Santacroce di Roma e scoperto dal professor Giuseppe Tommasetti. Si tratta di una «relazione, dettata da Domenico Gerosolimitano e da lui sottoscritta, sopra alcuni santuari di S. Giorgio, che egli aveva veduti in Oriente» ${ }^{16}$, relazione che il Guidi annunciava di prossima pubblicazione da parte del professor Alberto Parisotti ${ }^{17}$. Purtroppo allo stato attuale delle indagini, non risulta che il Parisotti abbia mai edito tale relazione e per di più l'originale, che apparteneva al fondo Santacroce depositato presso l'Archivio di Stato di Roma, risulta a tutt'oggi irreperibile ${ }^{18}$. Il Guidi inoltre, sulla scorta delle parole di Domenico, che nell'autobiografia scriveva: «nell'età mia circa quaranta al 1593 venni alla Santa fede Christiana» ${ }^{19}$, ne poneva la data di nascita

${ }_{15}$ Cfr. I. GuidI, «Domenico Gerosolimitano», in Festschrift zum siebzigsten Geburtage A. Berliner's (Frankfurt a.M. 1903) pp. 176-179: p. 177. Concordano con lui A. M. RABELLo, «Domenico Gerosolimitano», in Encyclopaedia Judaica (Jerusalem 1972) vol. VI col. 158; F. PARENTE, «La Chiesa e il "Talmud"», in Storia d 'Italia, Annali 11: Gli ebrei in Italia, I, Dall'Alto Medioevo all 'età dei ghetti, a c. di C. VIVANTI (Torino 1996) pp. 521-643: p. 605 nota 178. Secondo altri la data di nascita sarebbe il 1550. Cfr. G. BARToloccI, Bibliotheca Magna Rabbinica (Romae, ex typographia Sacrae Congregationis de Propaganda Fide 1678) pars secunda pp. 281-283: p. 281; W. POPPER, «Dominico Irosolimitano or Hierosolymitano», in The Jewish Encyclopedia 12 voll. (New York - London 1901-1906) vol. IV p. 635; Index Bio-Bibliographicus Notorum Hominum, edd. O. et W. ZELLER (Osnabruck 1992) vol. LVII p. 91. Risulta pertanto palesemente infondata la data 1530 proposta da Renata Segre (cfr. R. Segre, «La Controriforma: Espulsioni, conversioni, isolamento», in Storia d'Italia, Annali 11 pp. 707-778: p. 756).

16 Guidi «Domenico Gerosolimitano» p. 176.

17 Lo stesso Parisotti ne dava notizia in un suo scritto (cfr. A. PARISorti, «Intorno alla leggenda di S. Giorgio», Bollettino della Società Filologica Romana 5 (1903) pp. 33-39: p. 39.

${ }^{18}$ Ricerche da me compiute nel fondo Santacroce presso l'Archivio di Stato di Roma, dal cui catalogo manoscritto appariva l'esistenza della relazione in oggetto, hanno permesso di verificare che tale manoscritto non è più reperibile nei depositi dell'Archivio di Stato di Roma. Cfr. Archivio di Stato di Roma, Archivio Santacroce, Inventario N. 47, p. 204, Monumenti di Cristianità e dell'Ordine de Cavalieri di S. Giorgio esistenti in Costantinopoli e nel Dominio Turco registrati in forma autentica, ms. 94.A.31 (mancante).

${ }^{19}$ Guidi «Domenico Gerosolimitano» p. 176. 
verso il 1552, tuttavia, sulla scorta dell'atto di battesimo reperito nell'Archivio dei Catecumeni di Venezia, ritengo che il 1555 sia più plausibile.

Dalla scarna documentazione dei Catecumeni ${ }^{20}$, apprendiamo anche che Domenico, all'indomani della sua conversione, il 9 agosto 1593, collaborava con meser Eusebio Renati ${ }^{21}$ alla «revision delli libri hebrei», al controllo cioè e alla censura dei testi ebraici prima e dopo la stampa ${ }^{22}$. L'azione di Domenico in tale delicato settore, anche se non sappiamo per quanto tempo ebbe a svolgerla e se la sua fu una funzione ufficiale o solo una collaborazione con i censori a ciò deputati, venne così ad inserirsi nella prassi del controllo preventivo dei testi ebraici -eccetto il Talmud e i suoi commenti che restavano proibiti- da parte di due ebraisti, prassi che a Venezia era stata stabilita dagli Esecutori contro la bestemmia nel $1559^{23}$. Domenico si trovò ad operare in un contesto di rinascita dell'editoria ebraica: l'ultima decade del Cinquecento vide infatti non solo affluire a Venezia una gran quantità di libri proibiti ma fu anche un periodo di nuovo impulso per la tipografia in ebraico perché la Repubblica disattese sistematicamente i decreti pontifici che erano stati emessi contro di essa ${ }^{24}$.

I nuovi documenti da me individuati ci permettono quindi di anticipare al 1593 l'attività di Domenico quale censore di libri ebraici ${ }^{25}$

20 Cfr. AIRE, Catecumeni, CAT B 4, c. $23 \mathrm{v}$.

21 Il neofito Eusebio Renato o Renati, da ebreo Bonaventura, che, per alcune volte, fu rettore della Casa dei Catecumeni a Venezia tra il 1580 e il 1590 , incaricato del controllo dei libri ebraici a Venezia, che compare in alcuni processi del S. Uffizio veneziano. Cfr. B. Pullan, Gli Ebrei d'Europa e l'Inquisizione a Venezia dal 1550 al 1670 (Roma 1985) p. 520; Processi del S. Uffizio di Venezia contro Ebrei e Giudaizzanti (1548-1743), a cura di P. C. Ioly Zorattini, 13 voll. (Firenze 1980-1997) voll. VI p. 63 nota 1, VII p. 8 nota 5, VIII pp. 212-213.

22 Sui rapporti tra editoria veneziana e Inquisizione e sul problema della censura a Venezia cfr. P. F. GRENDLER, The Roman Inquisition and the Venetian Press: 1540-1605 (Princeton 1977; trad. ital. Roma 1983, edizione dalla quale si cita); P. C. Ioly ZoRATTINI, «Censura e controllo della stampa ebraica a Venezia nel '500", in Manoscritti, frammenti e libri ebraici nell Ttalia dei secoli $X V-X V I$, a c. di G. Tamani e A. Vivian (Roma 1991) pp. 115-127; M. Jacoviello, «Proteste di editori e librai veneziani contro l'introduzione della censura sulla stampa a Venezia (1543-1555)», Archivio Storico Italiano 151 (1993) pp. 27-56; P. C. Ioly Zorattini, «Il S. Uffizio di Venezia e il controllo della stampa ebraica nella seconda metà del Cinquecento", in La censura libraria nell'Europa del secolo $X V I$, a c. di U. Rozzo (Udine 1997) pp. 127-146.

23 Cfr. Grendler Venetian Press p. 201.

24 Cfr. Grendler Venetian Press pp. 355-356.

25 Il Popper proponeva come data d'inizio dell' attività censoria di Domenico a Venezia il 1594. Cfr. PopPER «Dominico Irosolimitano» p. 76. 
e probabilmente uno dei primi testi che egli ebbe modo di controllare fu il Mif'alöth Elohim di Isaac Abravanel, opera che venne edita a Venezia da Giovanni di Gara nel $1592^{26}$ e fu oggetto di una denuncia al S. Uffizio ${ }^{27}$, il 9 giugno 1592, da parte del sacerdote Sebastiano Tagliapietra, lettore di ebraico nel Seminario patriarcale di Venezia e revisore dei libri ebraici per conto del locale S. Uffizio ${ }^{28}$. Sebbene dagli atti del S. Uffizio non compaia altro che la denuncia del Tagliapietra e il costituto dell'editore, grazie alla scoperta presso la National and University Library di Gerusalemme di due copie del Mif'aloth, una purgata ed una nella versione originaria, siamo in grado di sostenere che l'opera fu effettivamente censurata anche se alcune copie dovettero in qualche modo sfuggire alla purga censoria. $\mathrm{E}$ che Domenico, sebbene il suo nome non compaia nei documenti del S. Uffizio veneziano, abbia avuto parte nel controllo del volume ce lo afferma egli stesso nel Sefer ha-Zikkūk (Canon purificationis seu liber expurgationis) ${ }^{29}$, un manuale censorio per i libri ebraici di cui egli era tra i compilatori, quando ricorda che al tempo del suo soggiorno veneziano si era accesa tra i censori una disputa sulla liceità di un brano del Mif'alöth concernente la sorte dei peccatori, disputa in cui il suo parere era stato determinante per la censura di tale passo: «Su questo sorse una gran controversia quando ero a Venezia e in base al mio parere lo condannarono» ${ }^{30}$.

${ }^{26}$ Su quest'opera di Abravanel cfr. I. E. BARzILAY, Between Reason and Faith: Antirationalism in Italian Jewish Thought, 1250-1650 (The Hague - Paris 1967) pp. 74, 80, 88-91; B. Netanyahu, Don Isaac Abravanel Statesman and Philosopher (Philadelphia 1968) pp. 66, 80, 297-298, 300-301, 304.

${ }^{27}$ Cfr. P. C. Ioly Zorattini, «Il Mif'aloth Elohim di Isaac Abravanel e il Sant'Uffizio di Venezia», Italia 1 (1996) pp. 54-69.

${ }^{28}$ Su di lui cfr. Ioly Zorattini «S. Uffizio di Venezia» p. 141 e nota 65.

${ }^{29}$ Sul Sefer ha-Zikkūk cfr. J. Fürst, Bibliotheca Judaica: Bibliographisches Handbuch der gesammten jüdischen Literatur mit Einschluss der Schriften über Juden und Judenthum und einer Geschichte der jüdischen Bibliographie, 3 voll. (Leipzig 1849-1863) vol. III pp. XVIII, 183; Cataloghi dei Codici orientali di alcune Biblioteche d'Italia (Firenze 1878) pp. 365-370; A. BerLiner, Censur und Confiscation hebräischer Bücher im Kirchenstaate (Frankfurt a.M. 1891) p. 9; G. SACERDOTE, «Deux index expurgatoires des livres hébreux», Revue.des Études Juives 30 (1895) pp. 257-283; N. PORGES, «Der hebräische Index expurgatorius "Sepher haZikkuk"», in Festchrift ... Berliner (Frankfurt a.M. 1903) pp. 272-295; W. PoPPER, The Censorship of Hebrew Books (rist. New York 1969) pp. IX-X, XXVIII, 81 e sgg., 99, 101-103, 119, 126; N. S. Leibovitz, Rabbi Abraham Saba U-sefarav Seror Ha-mor Ve Eshkòl Ha-kòfer (New York 1936) pp. 9-10.

${ }^{30}$ IOLY ZORATTINI «Mif'aloth» p. 63. 
Poco di più si sa sul suo soggiorno veneziano in questo tempo, da un altro documento dei Catecumeni si apprende che Domenico sarebbe partito da Venezia verso l'Italia l'11 febbraio 1594 con le credenziali fornitegli dalle autorità ecclesiastiche per «cercar di metter in stampa la sua opera», un riferimento oscuro ad uno scritto attualmente non identificabile che egli avrebbe composto o che avrebbe avuto con sè durante il suo soggiorno nella città della laguna.

Tuttavia prima del suo trasferimento in Lombardia, dove lo troviamo nel $1595^{31}$, Domenico è citato come presente a Venezia verso la fine dello stesso 1594 nel memoriale inviato, il 12 dicembre 1594, al S. Uffizio romano da un portoghese residente in quegli anni in Italia, fra' Crisostomo o Cristoforo della Visitazione procuratore generale dei Cistercensi ${ }^{32}$. Questo memoriale offre un interessante spaccato sull'opera di controllo da parte dell'Inquisizione romana dei Giudaizzanti iberici che erano tornati più o meno esplicitamente alla pratica dell'Ebraismo in Italia ed è una spia preziosa per delineare alcuni aspetti della collaborazione tra le autorità inquisitoriali italiane e gli ambienti ecclesiastici iberici, in questo caso particolare portoghesi ${ }^{33}$. Esso evidenzia anche un aspetto fino ad oggi sconosciuto della vita di Domenico: i suoi rapporti con i delatori del S. Uffizio romano nei confronti dei Giudaizzanti lusitani residenti a Venezia negli ultimi anni del Cinquecento. Nel memoriale fra' Cristoforo insisteva affinché la Congregazione romana del S. Uffizio facesse venire a Roma «meser Dominico rabbino che fu constantinopolitano» ${ }^{34}$ con

31 Dal frammento dell'autobiografia di Domenico contenuta nella suddetta relazione, edito dal Guidi, risulta che il Nostro aveva soggiornato a Milano dove aveva insegnato l'ebraico e svolto attività censoria nella biblioteca del cardinal Federico Borromeo, arcivescovo di Milano dal 1595 (cfr. P. P. BoscA, De origine et statu Bibliothecae Ambrosianae [Mediolani, Typis Ludovici Montiae MDCLXXII] p. 15; Aldo Luzzatro, Hebraica Ambrosiana: Catalogue of Undescribed Hebrew Manuscripts in the Ambrosiana Library [Milano 1972] pp. 3-4). Successivamente Domenico si trasferì a Mantova dove, dal 1595 al 1597, fu attivo come censore di libri ebraici (cfr. S. Simonsohn, History of the Jews in the Duchy of Mantua [Jerusalem 1977] pp. 690-691; PARENTE «Talmud» p. 605 nota 178). Dal 1598 lo troviamo a Ferrara (cfr. Popper Censorship pp. 93, 141) e poi a Roma dove fu lettore di ebraico nel Collegio dei Neofiti e dove scrisse alcune opere e tradusse in ebraico il Nuovo Testamento e dove morì dopo il 1621 (cfr. GuIDI «Domenico Gerosolimitano» p. 178). Erroneamente il Parente data l'anno di morte al 1612 (cfr. Parente «Talmud» p. 605 nota 178).

32 Su di lui cfr. P. C. Ioly Zorattini, «Un profilo del Marranesimo alla fine del '500: la denuncia al S. Uffizio romano di fra' Zaccaria da Lisbona» (in corso di stampa nel volume in memoria del prof. I. S. Révah).

33 Cfr. Ioly Zorattini Processi: Appendici, vol. XIII pp. 218-223.

34 Ioly Zorattini Processi: Appendici, vol. XIII p. 221. 
qualche motivo pretestuoso perché la sua venuta non destasse sospetti tra i Giudaizzanti veneziani in quanto l'ex rabbino poteva rivelarsi un testimone prezioso per svelare all'Inquisizione la doppia identità di diversi Marrani residenti nella capitale della Serenissima che egli aveva avuto modo di conoscere di persona.

L'importanza di Domenico nella lotta contro gli apostati veniva riconosciuta anche da un altro religioso portoghese, il quale fornisce ulteriori dati che arricchiscono le nostre conoscenze sulla vita del Gerosolimitano: mentre si trovava a Costantinopoli come medico di Murad III (1574-1595) ${ }^{35}$ sembra avesse svolto un ruolo attivo in favore della riconversione all'Ebraismo dei Marrani iberici. Infatti nel suo memoriale, presentato il 3 novembre 1593 al S. Uffizio romano, fra' Zacarias da Lisbona, un cappuccino che nell'ultimo decennio del secolo svolse un'intensa opera di controllo sui rapporti tra i Giudaizzanti residenti in Italia e i loro corrispondenti iberici ${ }^{36}$, segnalava tra un gruppo di Giudaizzanti portoghesi che vivevano ormai come Ebrei nel ghetto di Venezia tre fratelli originari di Lisbona, Garcia, Manoel e Alvaro Pimintel o Pimentel e informava come quest'ultimo, Alvaro, quattro anni prima, si fosse trasferito a Costantinopoli per farsi ebreo ${ }^{37}$, ed era stato raccomandato a «meser Domenico», «medico del Turco», il quale sembra essere legato ai Pimentel da vincoli di parentela, affinché lo circoncidesse ${ }^{38}$. Alvaro aveva quindi fatto ritorno a Venezia, dove aveva ripreso a vivere formalmente come cristiano fuori del ghetto e qui, sentendo compromessa la sua sicurezza dalla scomoda presenza in città del Gerosolimitano che, proprio a Costantinopoli, gli era stato maestro nella sua riconversione alla religione dei padri, lo aveva minacciato di dure ritorsioni se avesse avuto l'ardire di denunciarlo. Evidentemente, come nota fra' Zacarias, gli Ebrei avevano reagito duramente alla conversione di un rabbino tenuto in grande considerazione per sapienza e probità di vita dai correligionari che erano soliti offrirgli

${ }^{35}$ Cfr. A. Galanté, Médecins Juifs au service de la Turquie (Istanbul 1938) p. 11 (rist. Histoire des Juifs de Turquie, 9 voll. [Istambul s.a.] vol. IX p. 87); N. Koren, Jewish Physicians: A Biographical Index (Jerusalem 1973) p. 42. Il Koren sostiene erroneamente che Domenico Jerushalmi «was baptized in Rome». Sulla comunità ebraica di Istambul alla fine del Cinquecento cfr. S. Yerasimos, «La communauté juive d'Istambul à la fin du XVIe siècle», Turcica 27 (1995) pp. 101130 , che non accenna al Nostro.

36 Su di lui cfr. Ioly Zorattini «Marranesimo».

37 Cfr. Ioly Zorattini Processi vol. XIII p. 197 e nota 48.

${ }^{38}$ Cfr. Ioly Zorattini Processi vol. XIII pp. 204-205. 
«lemosine» perché «pregasse Iddio per essi». Infine, la parentela coi Pimentel riferita da fra' Zacarias ci permette di aggiungere un altro tassello alla scarna biografia del Gerosolimitano. Il rabbino Samuel Vivas che al momento del battesimo si era presentato a Venezia come ebreo levantino, era invece probabilmente di origine iberica, forse portoghese ${ }^{39}$ o discendeva da quegli Ebrei spagnoli che all'indomani dell'editto di espulsione dei Re Cattolici nel 1492 si erano trasferiti nel vicino Portogallo ${ }^{40}$.

Non si hanno, allo stato attuale delle ricerche, altre notizie relative al periodo veneziano di Domenico Gerosolimitano ma non v'è dubbio che Venezia segnò, con la scelta della conversione, un momento di capitale importanza nella sua vita. Ci sfuggono anche i motivi che indussero il Nostro alla conversione ma non c'è dubbio che questa dovette essere profondamente motivata: ne è prova l'impegno successivo di Domenico come censore, come apologèta del Cristianesimo e come fautore della conversione degli antichi correligionari; al riguardo ricordiamo il suo Ma 'ayan Gannim (Fons hortorum) sui fondamenti della fede cristiana e la sua traduzione integrale in ebraico del Nuovo Testamento ${ }^{41}$. Domenico si dedicò infine all'insegnamento dell'ebraico nel Collegio dei Neofiti ${ }^{42}$, tra le cui mura trascorse gli ultimi anni della sua vita chiudendo così quella carriera di revisore e censore della stampa ebraica che aveva iniziato proprio nella città in cui era avvenuta la sua conversione, quella Venezia che, se pur in maniera più ridotta, era ancora, alla fine del Cinquecento, il principale emporio italiano per la produzione e il commercio del libro ebraico ${ }^{43}$.

${ }^{39} \mathrm{Nel}$ corso del Quattrocento il cognome Vivas è attestato in Portogallo. Cfr. M. J. Pimenta Ferro Tavares, Os Judeus em Portugal no século XV, 2 voll. (Lisboa 1982-1984) vol. I pp. 33, 62, 132, 223, 285, 298, 356, 408.

${ }^{40}$ Sull'arrivo dei Marrani e degli Ebrei spagnoli in Portogallo a seguito dell'espulsione del $1492 \mathrm{cfr}$. I. S. RÉVAH, «Les Marranes portugais et l'Inquisition au XVIe siècle», in The Sephardi Heritage, ed. by R. D. BARNETT, 2 voll. (London 1971-1989) vol. I pp. 479-526: pp. 482-483; M. J. Pimenta Ferro Tavares, Judaísmo e Inquisição (Lisboa 1987) pp. 23 sgg.

${ }^{41} \mathrm{Si}$ veda al riguardo la sua traduzione in ebraico del Nuovo Testamento. Cfr. BARTOLOCCI Bibliotheca pars II p. 282.

${ }^{42}$ Sul Collegio dei Neofiti fondato a Roma da Gregorio XIII cfr. K. HoffmanN, Ursprung und Anfangstätigkeit des ersten päpstlichen Missioninstituts: Juden-und Mohammedanermission im XVI. Jahrhundert (Münster 1924); W. H. RUDT DE Collenberg, «Le baptême des Juifs a Rome de 1614 a 1798 selon les registres de la "Casa dei Catecumeni" », Archivum Historiae Pontificiae 24 (1986) pp. 91-231.

${ }_{43}$ Tra il 1592 e il 1600 vennero editi a Venezia circa quarantacinque testi in ebraico. Cfr. Grendler Venetian Press p. 356. 


\section{APPENDICE DOCUMENTARIA}

ASPV, Battesimi dei Catecumeni, reg. 1 «Battesimi principia 1590, 3 marzo finisse 1594 ultimo marzo», cc. $14 \mathrm{v}-15 \mathrm{r}$.

[1593] $]^{\text {14v }}$ Adì primo luglio venne Samuel Vivas hebreo levantin da Gierusalem, de anni XXXVIII in circa, in hebreo chiamato chaccham, che in italiano dicemo dottor, per battezzarsi e fu accettado dalla magnifica Congregation.

115r Alli VI agosto MDLXXXXIII.

Il contrascritto Samuel fu battizzato per il reverendo et illustrissimo monsignor patriarcha de Venetia in chiesa de Santi Gioanni e Paulo et li fu posto nome Dominico. Li santoli suoi furono il reverendissimo monsignor arcivescovo di Spalato, il reverendissimo monsignor vescovo di Milopotomo.

Il soprascritto Dominico, adì 8 settembre 1593, fu chresmato in Patriarchato. Il suo compare fu il clarissimo signor Hieronimo Corner governator di questa Casa.

Adì 11 febraro 1594 partite da Venetia per Italia con le sue fede per cercar di metter in stampa la sua opera, come disse.

\section{RESUMEN}

El artículo se ocupa de la identificación hebrea de Domenico Gerosolimitano, célebre censor de libros hebreos que trabajó en Italia entre fines del siglo XVI y los primeros veinte años del XVII. Gracias a haber descubierto en Venecia el acta de bautismo, el autor ha logrado identificar a Domenico Gerosolimitano con el rabino Samuel Vivas de Jerusalén y precisar la fecha exacta de su conversión al catolicismo (6 de agosto de 1593). El estudio arroja además nueva luz sobre la estancia en Venecia de Domenico y sobre sus inicios como censor de libros hebreos.

\section{SUMMARY}

In this article, the author provides the Hebrew name of Domenico Gerosolimitano, a famous ecclesiastical censor of Hebrew books who lived in Italy from the end of the sixteenth through the second decade of the seventeenth centuries. Based on the discovery of his baptismal act, recorded in Venice, Domenico Gerosolimitano's Hebrew name was Rabbi Samuel Vivas, of Jerusalem. The aforementioned act determines the exact date of his conversion to Catholicism, as 6 August, 1593. The author presents new information concerning Domenico's life in Venice as well as his activities as censor of Hebrew books. 